\title{
A Study on the Change of Career Values of Hotel Management Students -- Based on Cognitive Dissonance Theory
}

\author{
Chao Chia-Wei ${ }^{1, a^{*}}$, Zhong Zi-Sheng ${ }^{2, b}$ \\ ${ }^{1}$ Toruism and Historical Culture College, Zhaoqing University, Zhaoqing, Guangdong, China \\ 2 Hospitality Management department Student, School of Tourism and Historical Culture, Zhaoqing University, \\ Zhaoqing, Guangdong, China \\ a*1991150933@qq.com \\ b1124357827@qq.com
}

\begin{abstract}
What is the reason why most hotel management students have fled the industry after internship? What factors affect their career values in the process of internship? Based on the theory of cognitive dissonance and the method of in-depth interview, the research conducted in-depth interviews with 8 interns of hotel management. The interviews were conducted in three sessions, namely, three periods before internship, internship and internship, and the duration of the interview ranged from October 2019 to March 2021.The data obtained from interviews will be analyzed through content analysis. The study found that the reason for the change in the value of employment values was that the information of the hotel industry and the hotel industry that they knew were in a low degree of agreement with expectations. In order to alleviate and change this situation, this study will provide students with personal and hotel management professional institutions and units to provide relevant feasible suggestions.
\end{abstract}

Keywords: Cognitive dissonance theory, Career values, In-depth interview, Content analysis

\section{因了解而分开？认知失调理论下酒店管理专业学生 的择业价值观的改变一一以实习生为例}

\author{
赵家伟 ${ }^{1, a *}$ 钟子胜 ${ }^{2,}$ b*
}

肇庆学院旅游管理与历史文化学院, 肇庆, 广东, 中国

2 肇庆学院旅游管理与历史文化学院酒店管理系毕业生, 肇庆, 广东, 中国

a*1991150933@qq.com

b1124357827@qq.com

\section{摘要}

多数酒店管理专业的学生在实习后纷纷逃离该行业, 这是为什么呢? 实习过程中是什么因素影响了其择业价 值观呢? 本研究以认知失调理论的视角并采用深度访谈法的研究方法进行资料收集，对 8 位酒店管理专业的 实习生进行面对面的深度访谈，访谈分三次进行，分别是实习前、实习中和实习后三个时间段，访谈时间跨度 为 2019 年 10 月至 2021 年 3 月，访谈获得的资料将通过内容分析法进行分析，研究结果发现影响其择业价值 观改变的原因是实习单位和其了解到的酒店行业的信息与其实习前所了解与期待的吻合度较低。为了缓解并 改变这个局面, 本研究将给学生个人、开展酒店管理专业的院校和单位提供相关的可行性的建议。

关键词: 认知失调理论，择业价值观，深度访谈，内容分析 


\section{1. 前言}

当代大学生面临着巨大的压力, 为了更好的就业, 大学生找与自己在读专业相关的职业无疑更具竞争 力。但根据调查, 2017 年毕业生近四成毕业生就业岗 位与专业不对口 [1]。随着我国酒店的规模的扩大以 及等级的提高, 酒店对员工的素质要求也越来越高, 酒店管理专业的学生具有一定的理论素养以及实践 能力。相对于行业规模的急速扩大, 我国酒店行业的 从业人员的数量增幅却呈缓慢上升趋势。2000 年平均 每间客房的员工数量为 1.186 人/间, 而到 2019 年已 下降至 0.999 人/间 [2]。越来越少人选择从事酒店 行业, 人才的大量流失已经成为酒店行业面临的主要 问题。酒店人才补给的不足, 酒店人才严重的离职率 很大程度上取决于求职者的职业规划。过往研究发现, 在职业探索后期的酒店专业实习生对所实习酒店以 及在酒店行业发展前景的评价会有很大程度上影响 其未来的职业规划 [3]。这不得不让我们思考实习过 程中是什么因素影响实习生今后的职业规划? 本研 究将针对这问题进行探究并给出答案。

\section{2. 文献综述}

认知失调是指在一般情况下, 个体对于事物的态 度以及态度和行为之间是相互协调的, 如果不协调, 就会产生不和谐的认知, 即认知失调 [4]。Telci、 Maden 与 Kantur 认为认知失调理论是关注认知之间 的关系, 认知是人们对自己的行为、态度、感知或者 感觉拥有的知识的组成部分 [5]。阳海英等人指出认 知失调是指当个体觉察到自己的各种态度之间或者 态度与行为之间存在不一致的情况时, 产生的一种不 愉快的内部状态 [6]。综上, 本研究认为认知失调是 指主体对客体的综合主观认知与客体自身的客观实 际情况吻合度低, 从而在心理上产生一种抗拒的心理 状态。过往研究也指出对大学生就业中的认知失调现 象的研究中提出认知失调现象主要原因是学生对工 作内容失望, 对自我价值的贬低以及对受教育的怀疑 等, 因实际情况与理想出现矛盾, 认知失调也就随之 而来 $[7]$ 。那翠翠认为旅游管理本科生之所以会出现 专业失调, 主要是因为在选择专业前没有深入了解这 专业, 其次就是受到以往所接触的, 了解的对这专业 的观点的影响的结论 [8]。

在择业价值观部分, 袁贵仁提出价值取向是人们 在一定场合以一定方式采取一定行动的价值倾向 [9]。 过往研究也指出择业价值观包含了地域的选择、行业 的选择与价值目标等因素, 反映了主体对于行业以及 个人价值的实现的认知水平, 择业价值观也是影响人 们择业的重要因素 [10]。综观上述, 本研究把择业 价值观定义为个体对其职业规划的目的、意义以及标 准等的根本的看法和态度, 是个体价值观在职业规划 上的集中体现。顾凡和师听强认为近年来大学生就业 日趋严峻, 大多数专业设置与市场严重脱节, 就业市 场不完善以及大学生的择业观的不成熟等成为影响
大学生择业的重要因素 [11]。综上, 择业价值观受学 生个人、家庭、学校、市场环境以及政府等因素影响, 本研究将从这几个方面出发, 研究择业价值观受这几 个方面的影响后的改变情况。

实习顾名思义就是在实践中学习 [12]。都昌满认 为实习是高校致力于培养学生的实践能力, 工作能力, 确保人才培养目标实现的重要教学环节, 同时也是学 生增强就业能力, 提高自身竞争力的重要手段 [13]。 综上研究, 本研究定义实习为学校为了掌握大学生知 识掌握情况, 提高学生实践能力以及适应社会的能力 的一教学环节。龚敏在研究中得出实习满意度是影响 其择业意向的重要因素, 在实习后, 择业意向一般都 会发生变化, 实习满意度的提升是学生择业价值观是 否健康的重要影响因素 [14]。因此, 本研究选择以大 学生的实习作为切入点, 深入研究探讨基于认知失调 了理论下大学生择业价值观的改变情况。

\section{3. 研究设计}

本研究以认知失调理论为基础, 为了解实习生在 实习前、实习中与实习后在认知观念与心态上的改变, 进而厘清认知失调的因素, 对于实习前访谈目的主要 是了解学生尚未正式进入该行业之印象与认知, 实习 中访谈目的主要了解实习过程对于该行业的认知情 况, 实习后访谈主要目的为了解学生对于整体行业了 解与今后择业意愿, 依据不同目的设计问卷; 调查方 式为深入访谈或者电话访谈搭配录音为主, 后续形成 逐字稿, 给予受访者确认内容, 以求资料真实性与完 整性, 访谈时间为 2019 年 10 月至 2020 年 9 月, 横 跨时间一年，对象为酒店管理专业实习学生。

深入访谈法是质性研究中极其重要的收集方之 一, 具有很大灵活以 深入访谈法是质性研究中极其 重要的收集方之一, 具有很大灵活以 及对意义进行 诠释的空间 [15]。深度访谈法适用于了解复杂、抽 象的问 题, 这类深度访谈法适用于了解复杂、只有 通过交谈对所关心主深入探讨才能概括出其中的更 深层含义。深度访谈后, 本研究将采用内容分析法对 访谈所获得的资料进行分析, 藉以得到本研究结果。

\section{4. 资料分析}

本研究主要探讨基于认知失调理论下酒店管专 业的择价值观改变, 挑选的样本是酒店管理专业的学 生, 使用深度访谈法在实习前期、中期以及后对采 访象开展 3 次深度访谈 以索取本研究需要的资料信 息, 本研究采访到第 8 位访谈对象时发现采内容已达 饱和状态, 在内容编码方式上, 以 “受访人员 位学 生。以 “受访人员 编号 一行数” 进编码, 样本相关 介绍如表 1 所呈现。

表 1 访谈样本

\begin{tabular}{|c|c|l|c|}
\hline 编号 & 性别 & 实习单位 & 实习/部门岗位 \\
\hline $\mathrm{D} 1$ & 女 & 三亚 $\mathrm{Y}$ 酒店 & 前台接待员 \\
\hline
\end{tabular}




\begin{tabular}{|c|c|l|c|}
\hline D2 & 女 & 深圳 $\mathrm{E}$ 酒店 & 销售助理 \\
\hline D3 & 女 & 广州 $W$ 酒店 & 宴会 \\
\hline D5 & 女 & 广州 $W$ 酒店 & 总机接待 \\
\hline D7 & 女 & 东莞松山胡 $\mathrm{K}$ 酒店 & 宾客接待 \\
\hline D8 & 女 & 三亚 $\mathrm{A}$ 酒店 & 财务部 \\
\hline D9 & 男 & 深圳 L 酒店 & 西餐厅 \\
\hline D10 & 男 & 三亚 A 酒店 & 礼宾部行李员 \\
\hline
\end{tabular}

实习前的心态与期待, 对于初步踏入社会的大学 酒店管理专业实习生来说，对实习充满着憧憬，在访 谈中都提到了在未深入了解该行业的前提下对于该 行业的种种期待，包括工资、福利和部门氛围等。内 容提到虽然实习包住宿以及一日三餐, 但是实习地点 基本都在珠三角发达城市, 消费水平高, 同学们认为 实习工资相对偏低; 也期待转正工资能符合自己心理 预期, 同学们都还没接触过这个行业, 还没了解酒店 行业的工资详情, 但是因为实习工资低, 所以同学们 希望实习后如果有机会转正, 工资可以符合自己的心 理预期。同时，希望同事间能够和谐相处，对于出入 职场的学生来说, 除了在乎工资待遇外, 能否和同事 们和谐共处也是他们比较在乎的。在采访中, 受访者 都希望在和老同事可以像朋友一般相处, 希望部门氛 围能如他们所期待的那样; 如果有必要接受加班, 但 希望酒店给与相应的补贴, 以及希望实习后转正可以 从领班做起、期待能在五年内升到经理岗位。希望能 在半年的实习中有所收获与毕业后会从事相关行业 工作皆为八位受访者在实习前的意愿与认知情况。

[确实蛮低的, 就我觉得如果是自己找的实习的话, 会 相对比跟学校合作的酒店给的工资会高点。JD5-14

[恩, 转正的话, 我期待工资可以在 5000 6000 左 右。 $7 D 2-68$

[希望是那种额就是工作的时候是我们比较团结, 然后 互帮互助, 然后平时的话也可以轻松一点, 然后额大家可 以互相交流进步那种。7D7 -98

\section{[无偿加班的话肯定是不太能够接受的。]D9-76}

[额, 如果是说, 就是毕业后的正式工作的话, 我觉得, 起码应该是从领班开始吧]D7-25

[部门经理的话, 就是希望的话应该是五六年这样。差 不多这个时间我觉得是我自己比较希望得。]D10-94

[恩希望能学到一些额比较如果是能力方面的话, 就想 多提高一下我各方面的能力, 比如说沟通能力, 协调能力 这些。]D5 -92

实习中期的了解与认知, 受访者带着实习前的对 实习生活以及酒店行业的期待到实习单位实习, 所以 他们会有意识地去了解现实中的实习生活与该行业 的业态是否符合其期待。结果发现酒店会要求员工无 偿加班、才能无处可施、酒店福利、转正后岗位、工 作氛围与预期不符, 整体工作环境也没有想像中的高 大上、人才培养制度不完善、升职速度没有想像中的 快等认知失调产生, 在访谈过程中也得知有半数受访 学生还是有意愿在毕业后从事酒店行业工作, 另有半 数择已明确表示不会。
[像餐饮部门的话, 他如果你加一小一小时之内的, 他 好像都不给你算工资, 但是那些实习生又不得不听从上面 的安排]D5 -77

[就是你实习阶段能用的, 大学超过有挺多就是你实习 阶段能用的, 大学超过有挺多就是你实习阶段能用的, 大学超过有挺多就是你实习阶段能用的, 大学超过有挺多 就是你实习阶段能用 的, 大学超过有挺多不上的 JD10 58

[像我在部门的话, 就我了解而言, 我们酒店它的福利 是很挺少]D9-15

[额就我们酒店来说, 一般都是最基本的服务员开始做 起, 就没有从领班做起。7D10-81

实习后期的认知与择业价值观，经过六个月的实 习, 同学们对酒店相关信息有了初步解自己与酒店, 是否毕业后从事相关工作也有所定夺，内容包括无偿 加班制度导致身心疲㤂、前景虽好，但转正后工资过 低、工作氛围压抑与同事间关系不好、升职难时间又 长、培养制度未完善、没有提升自己的机会、觉得屈 才, 以及后续八位同学皆表示在毕业后不从事酒店行 业。

[现在就是慢的我们加班时间还挺多，也比较习惯了。 但还是不太能接受, 我对这点的话比较就个人持反。7D7 $-81$

[额会的, 因为如果说一直都没有新福利制度而只是无 偿加班话我觉得我可能不久就会离职了。7D8 -70

[额我部门有一个是正式生, 他进来可能不够一年或者 一年, 然后他转正后的工资是 3000 多, 感觉挺低的。虽然 包吃包住。7D5 -61

[会啊, 因为自己本身也不能接受了, 所以就很难在这 个行业继续做下去。毕基本上都要从基层员工做起。7D581

[恩, 不会选择再在酒店行业了, 经历过就好。不会 选择再在酒店行业了, 经历过就好。JD5 -48

[恩会让我想要逃离这个行业。]D7-65

[额，我应该不会再选择留在这个行业。]D8-36

\section{5. 结论与建议}

如果实习后, 酒店管理专业的学生纷纷逃离酒店 行业, 那实习的目的到底是什么呢? 其实, 专业实习 是学校为了了解学生的知识掌握情况、提高学生的实 践能力及社会适应能力的一个重要环节 [16]。在了解 过后, 学生会离开所学专业之行业是正常的现象, 但 既然选择必然是对于该专业有较大的兴趣, 然而, 在 大部分学生实习结束后转行, 这情况就值得深思, 也 给予本研究一个破口去对酒店实习过程进行追踪调 查、从而找出实习过程中影响同学择业价值观的改变 原因, 这是本研究的意义所在。

研究结果表明, 在实习中影响学生择业价值观改 变的主要原因是因为认知失调的产生, 这与过往研究 发现大学生因为对自身认识不足, 以为学历高就应该 
从事顶尖或中高阶工作，不应到基层工作，这是屈才 的社会现象，从而产生严重的失调情况，与自身择业 价值观产生严重影响的观点不谋而合，包括实习单位 工作内容、培养制度、工作氛围与个人发展等因素影 响相互印证 [17-18], 除了上述因素, 本研究也发现加 班补贴、岗位期待与升职年限三个因素也同样影响著 择业价值观。

根据研究得出的结果, 想要缓解局面, 建议从学 生、高校与用人单位三方面进行相关沟通。首先, 学 生应提前了解酒店等企业, 了解行业具体信息, 此外, 也切勿好高袀远, 摆正心态、脚踏实地、树立自信与 不要怀疑自己受教育得到的知识与能力; 学校部分择 是建议上课过程中, 教师于课堂上事实讲授酒店工作 的相关信息, 实践课程应更新或者顺应酒店的最新要 求, 并创造学生与企业接触或者面对面交流的机会, 让学生可以多方深入了解行业; 酒店企业部分则是要 履行诺言, 完善并落实福利制度, 并合理分配工作量, 给予实习生更多耐心, 并改善薪酬制度与晋升制度等, 让学生可以感受到酒店的前景。对于本科酒店管理专 业的学生纷想逃离这行现象, 不管是学生、学校或者 是用人单位都应该给予足够重视, 三方都应努力想办 法改善局, 从而保证专业对口率, 防止出现社会人才 浪费以及酒店行业的人才流失现象。

\section{REFERENCES}

[1] Xiong Y. Y.(2018) Research on the fit between College Students' employment and specialty under the background of "mass entrepreneurship and innovation". CO-Operative Economy \& Science, 592(17):154-156.

[2] China Tourism Research Institute. (2010) China hotel industry development report, 2009. China Tourism Press, Beijing.

[3] Wang X. Q. (2008) on the influence of hotel practice on career choice tendency of students majoring in Tourism Management. Journal of Sichuan Higher Institute of Cuisine, (04):66-71.

[4] Festinger L. (1957) Cognitive dissonance theory. Translated by Zheng Q. Q. Zhejiang Education Press, Hangzhou.

[5] Telci E E, Maden C, Kantur D. (2011)The theory of cognitive dissonance: A marketing and management perspective. Procedia - Social and Behavioral Sciences, 24:378-386.

[6] Yang H.Y., Zhang L. W., Hui B. H., Lu W. (2015) Cognitive dissonance and self-loss of college athletes: the preventive effect of self-affirmation. China Sport Science, 35(5): 29-37.

[7] Jiang C., Luo Y., Zhang H. Y. (2017) A study on the mental disorder in the grassroots employment of University Students. Higher Education Exploration,
(2): 105-111.

[8] Na C. C.(2018) Study on cognitive dissonance of undergraduate students in Tourism Management Major. Dalian: Master's thesis of Liaoning Normal University.

[9] Yuan G. R. (1999) Introduction to axiology. Beijing Normal University Press, Beijing

[10] Xue L. F. (2010) College Students' career psychology and career values education. Journal of Northeast Normal University(Philosophy and Social Sciences), (1):175-178.

[11] Gu F., Shi X.Q. (2009) Study on Influencing Factors and Countermeasures of College Student's Jobhunting Intentions. Heilongjiang Researches on Higher Education, (1):83-85.

[12] Xu D. F. (2010) Let internship add points to job hunting . Career Development, (z2):116-117.

[13] Du C. M.(2010) College Students' practice: problem analysis and Solutions. Research in Higher Education of Engineering, (5):144-149.

[14] Gong M.(2018) A study on graduation practice satisfaction and career choice intention of students majoring in Tourism Management -- a case study of Changsha University. Journal of Higher Education, (7):65-66+70.

[15] Chen X. M. (2000) Qualitative research methods and social science research. Educational Science Press, Beijing.

[16] Zhang Y.(2016) The influencing factors and coping strategies of vocational college students' career choice intention in the economic transition period. Journal of China University of Labor Relations, (5):19-25.

[17] Zhong J. F., Li P.(2018) Exploration and Analysis of hotel practice mode of tourism management majortaking Guangdong Ocean University Cunjin College as a example. Journal of Jilin TV \& Radio University, 203(11):74-75.

[18] Bai L.(2017) On the phenomenon of cognitive dissonance in college students' employment at the grassroots level. Journal of Jiamusi Vocational Institute, (11):267. 